\title{
Parasexual Genetics in Dictyostelium discoideum: Mitotic Analysis of Acriflavin Resistance and Growth in Axenic Medium
}

\author{
By K. L. WILliAMS, R. H. KESSIN* AND P. C. NEWELL \\ Department of Biochemistry, University of Oxford, Oxford $O X \mathrm{I} 3 Q U$
}

(Received I 2 February 1974; revised 6 May 1974)

\section{SUMMARY}

Drug resistant mutants of the slime mould Dictyostelium discoideum useful for mitotic genetic analysis have been studied. Mutations to acriflavin resistance are recessive and have been assigned to two genes (acr $A, \operatorname{acr} B)$ located in different linkage groups. One of these (acr $A$ ) confers resistance to the unrelated compounds methanol and thiabendazole. Ability to grow axenically (in the absence of bacteria) is determined by genes on two linkage groups, one carrying acr $A$ and the other an established temperature sensitive mutation. Preliminary results for two linkage groups show that the sequence of genes on a linkage group can be determined by analysis of homozygous drug resistant diploids, obtained by drug selection from diploids heterozygous for recessive drug resistance markers.

\section{INTRODUCTION}

During the life-cycle of the cellular slime mould Dictyostelium discoideum, amoebae differentiate in response to starvation to form stalk and spore cells. Because of the relative simplicity of this differentiation and the ease with which the organism can be manipulated, it has been extensively studied by developmental biologists. Despite the availability of numerous mutants affecting the development of $D$. discoideum, no means of genetic analysis was available until recently to complement the biological and biochemical studies to which the organism is well suited. A complete sexual cycle has been postulated, but is not elucidated (Clark, Francis \& Eisenberg, 1973; Erdos, Raper \& Vogen, 1973). Genetic studies have therefore been based on parasexual analysis (Sinha \& Ashworth, I969; Katz \& Sussman, 1972). The parasexual system exploits rare but spontaneous fusions of haploid, growthtemperature sensitive amoebae, differing in their temperature sensitive mutations, to form temperature resistant diploids. Once formed, these diploids can be maintained indefinitely. Haploid segregants may be obtained by including recessive drug resistance markers in the diploids, which makes possible the selection of drug resistant haploids arising through spontaneous haploidization. Because chromosomes assort randomly without a high frequency of genetic exchange between homologues (mitotic crossing-over) any genetic marker may be assigned to a linkage group. However, selection of the products of mitotic crossingover permits the order and relative distance between markers on a linkage group to be determined (Pontecorvo \& Kafer, I958).

Good chromosomal markers are rare in $D$. discoideum. Since a defined medium for growth has not yet been developed, auxotrophic mutants are not available. Recessive muta-

* Present address: Division of Biological and Medical Sciences, Brown University, Providence, Rhode Island 0291 2, U.S.A. 
tions affecting pigmentation are known (Sussman \& Sussman, 1963) and are useful for monitoring the stability of diploids. The use of these pigmentation markers to recover rare $\left(\mathrm{IO}^{-3}\right)$ haploids without preselection on drugs is not practical in $D$. discoideum, although visual selection of haploid segregants on the basis of recessive colour mutations is used routinely in Aspergillus nidulans (Pontecorvo \& Kafer, 1958). Hence other selective markers must be sought. Katz \& Sussman (I972) characterized cycloheximide resistance as a recessive marker located on linkage group I. Complementation and linkage studies in this laboratory with three strains each containing an independently isolated cycloheximide resistance mutation suggest that there is only one gene in $D$. discoideum conferring a high level of resistance to cycloheximide $(500 \mu \mathrm{g} / \mathrm{ml})$. In response to a suggestion by Dr E. R. Katz, acriflavin resistance has been studied as a source of further recessive resistance mutations. Such mutations have been characterized in Aspergillus nidulans (Roper \& Kafer, 1957). As a result we report two genes determining resistance to acriflavin located on linkage groups I and II ( $a c r B$ and $a c r A$ respectively).

For biochemical studies the growth of $D$. discoideum on bacteria is a serious disadvantage, so axenic strains have been isolated (Sussman \& Sussman, 1967). The isolation of such strains is very time-consuming and few have been reported (Schwalb \& Roth, 1970; Watts \& Ashworth, 1970; Loomis, 1971). For this reason we studied the genetic basis of axenic growth in the hope that the ability to grow axenically could be inserted into non-axenic strains (Williams, Kessin \& Newell, 1974). From cytological evidence, D. discoideum is thought to have seven linkage groups (Brody \& Williams, 1974). We report here the assignment of axenic genes to two of the seven linkage groups.

\section{METHODS}

Strains. The characteristics of the strains used are detailed in Tables I and 2. Nomenclature is based on that used in bacterial genetics (Demerec, Adelberg, Clark \& Hartman, 1966). Growth-temperature sensitive mutants (NP2 and NP3) of the axenic strain AX3 were isolated following treatment with $N$-methyl- $N^{\prime}$-nitro- $N$-nitrosoguanidine (Ralph Emanuel Ltd, Wembley) and subsequent selection involving incorporation of bromodeoxyuridine and killing by near u.v. light (Kessin, Williams and Newell, unpublished). Spontaneous acriflavin resistant mutants were selected on Aerobacter aerogenes growing on nutrient agar plates containing acriflavin (100 $\mu \mathrm{g} / \mathrm{ml}$, Sigma, neutral) or methanol ( $2 \%, \mathrm{v} / \mathrm{v}$, Analar, Fisons). These concentrations were used in all experiments.

Maintenance of stocks and growth conditions. Stocks of all strains were maintained as spores in silica gel at o to $5{ }^{\circ} \mathrm{C}$ (Perkins, 1962). Strains being used routinely were maintained clonally in association with $A$. aerogenes on $\mathrm{I} \cdot 5 \%$ (w/v) nutrient agar plates at $22{ }^{\circ} \mathrm{C}$, and cloned weekly (Sussman, I966).

Formation of diploids. When two growth-temperature sensitive haploid strains were to be fused to form a temperature resistant diploid, $10^{5}$ to $3 \times 10^{5}$ amoebae of each strain were plated on separate nutrient agar plates in association with $A$. aerogenes. The amoebae were harvested as they cleared the $A$. aerogenes after approximately $48 \mathrm{~h}$ at $22{ }^{\circ} \mathrm{C}$, washed free of bacteria, and treated as described previously (Williams et al. 1974). Diploids appeared at the restrictive temperature $\left(27^{\circ} \mathrm{C}\right)$ at a frequency of about $\mathrm{I}$ in $10^{5}$ amoebae plated.

Selection of haploid segregants. Haploids were selected by plating $5 \times 10^{4}$ diploid amoebae, which were heterozygous for a particular drug resistance marker, together with $A$. aerogenes on nutrient agar containing the drug. The concentrations of inhibitor used in the nutrient agar were: cycloheximide (Sigma), $500 \mu \mathrm{g} / \mathrm{ml}$; acriflavin, I00 $\mu \mathrm{g} / \mathrm{ml}$; methanol, $2 \%$ (v/v). 


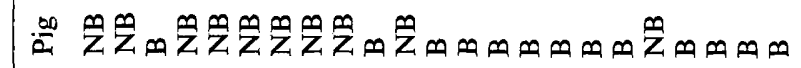

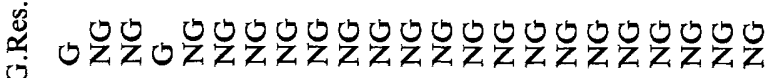

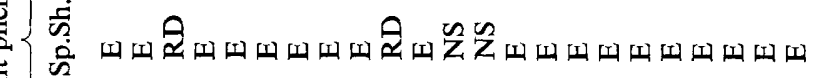

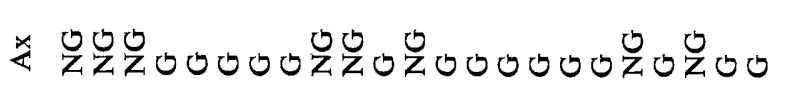
o

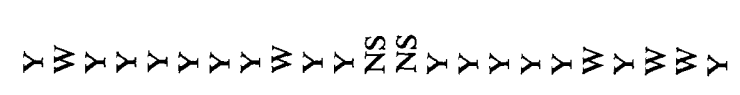

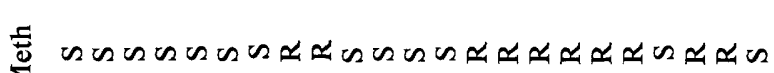

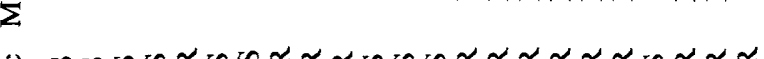

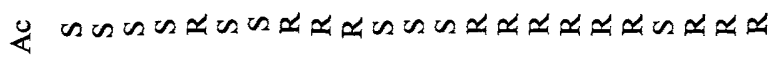

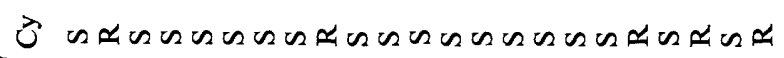
8

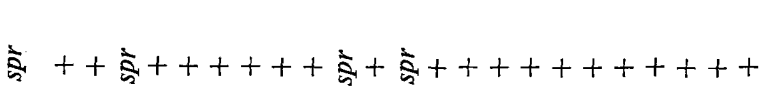

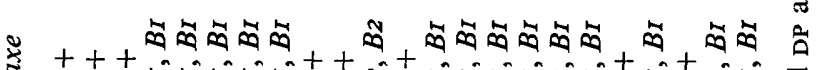

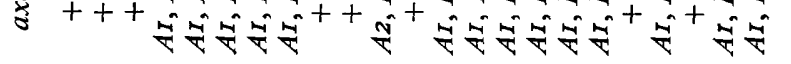

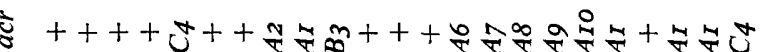

仓ั้ $+\not{7}+++++7+++++++++7+z+7$

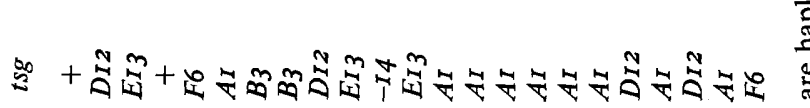

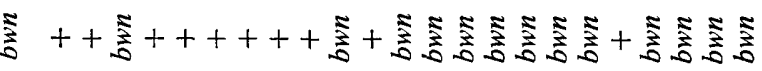

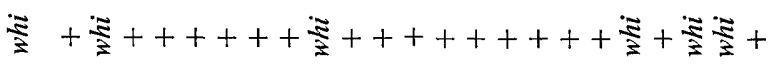

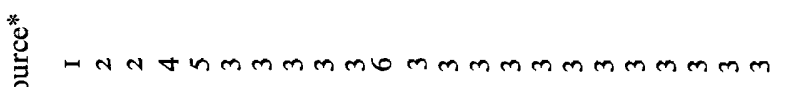
굴

$\dot{0} \dot{\vec{g}}$

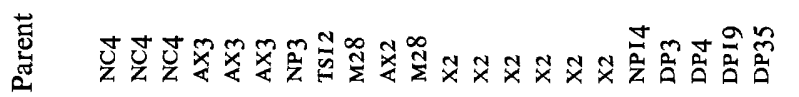

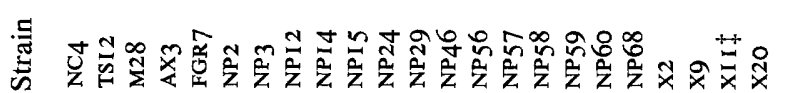


Table 2. Description of the diploid strains of D. discoideum used

All of the diploid strains were derived in this laboratory. All mutant alleles detailed here are recessive, so the genotype and phenotype of diploids can be determined from Table $\mathrm{I}$.

$\begin{array}{lccc}\text { Strain } & \text { Haploid parents } & \text { Strain } & \text { Haploid parents } \\ \text { DP2 } & \text { TSI2/NP2 } & \text { DP35 } & \text { X9/FGR7 } \\ \text { DP3 } & \text { M28/NP2 } & \text { DP40 } & \text { X20/TSI2 } \\ \text { DP4 } & \text { M28/NPI4 } & \text { DP44 } & \text { TSI 2/NP56 } \\ \text { DP5 } & \text { NPI5/TSI2 } & \text { DP45 } & \text { NPI4/NP57 } \\ \text { DP6 } & \text { NPI4/NPI5 } & \text { DP46 } & \text { NPI4/NP59 } \\ \text { DP8 } & \text { NP3/X9 } & \text { DP47 } & \text { NPI4/NP60 } \\ \text { DP19 } & \text { NP46/NPI4 } & \text { DP49 } & \text { TSI } / \text { NP59 } \\ \text { DP30 } & \text { X9/NP24 } & \text { DP54 } & \text { NP68/NP56 } \\ \text { DP32 } & \text { TSI 2/NPI2 } & \text { DP55 } & \text { NP68/NP58 } \\ \text { DP33 } & \text { X9/NPI2 } & \text { DP59 } & \text { TSI 2/NP58 } \\ \text { DP34 } & \text { NP29/NPI2 } & & \end{array}$

The plates were prepared by adding filter-sterilized inhibitor to molten agar immediately before pouring. After 4 to 5 days haploids and homozygous diploids resistant to the inhibitor were observed. These were differentiated on the basis of spore size and subsequent segregation of genetic markers (Katz \& Sussman, 1972). Cycloheximide was used at a higher concentration $(500 \mu \mathrm{g} / \mathrm{ml})$ than that used previously $(300 \mu \mathrm{g} / \mathrm{ml}$; Katz \& Sussman, 1972) since some axenic strains showed a partial resistance to cycloheximide at $300 \mu \mathrm{g} / \mathrm{ml}$.

In some cases haploid segregants were selected from heterozygous axenic/non-axenic diploids after prolonged incubation in axenic medium. Ability to grow axenically is a recessive character (Williams et al. 1974). Such haploid segregants were not used in assigning markers to linkage groups since many of them may have been of clonal origin.

Characteristics of haploid segregants. Clones appearing on inhibitor plates were picked with a sterile toothpick on to nutrient agar plates previously spread with $A$. aerogenes. Because the haploidization procedure is a single colony isolation method, further single colony isolation was generally not necessary. Occasionally, mixed cultures were observed which when analysed invariably proved to be a mixture of haploids and diploids and could be purified by single colony isolation. Colour markers (brown pigment production, white or yellow spores) and ploidy (on the basis of spore size) were established. The ability to grow at $27^{\circ} \mathrm{C}$ (restrictive temperature) and resistance to other inhibitors were determined by transferring with toothpicks on to appropriate agar plates spread with $A$. aerogenes.

Screening for axenic growth. Amoebae were transferred directly into I $\mathrm{ml}$ axenic medium (Watts \& Ashworth, 1970) plus $250 \mu \mathrm{g}$ dihydrostreptomycin sulphate (Sigma)/ml contained in sterile multiple-welled tissue culture dishes (Linbro FB-I6-24-TC). It was possible to pick amoebae directly from bacterial plates into axenic medium because the bacteria (streptomycin sensitive $A$. aerogenes) were regularly cloned. The culture dishes were shaken in the dark at 150 cycles/min at $22{ }^{\circ} \mathrm{C}$, and inspected with an inverted microscope (Olympus, Model CK, Tokyo, Japan) on at least two occasions approximately 3 and 7 days after inoculation. Axenic growers, defined as growing to more than $2 \times 10^{6}$ amoebae $/ \mathrm{ml}$ at stationary phase, were easily observed. In some cases axenic growth was confirmed in 25 or $125 \mathrm{ml}$ flasks, containing 5 or $25 \mathrm{ml}$ of axenic medium shaken at $\mathrm{I} 50 \mathrm{cycles} / \mathrm{min}$ at $22{ }^{\circ} \mathrm{C}$. 
Table 3. Dominance and complementation analysis of acriflavin resistance mutations

Either two acriflavin resistant haploids, or an acriflavin resistant and acriflavin-sensitive haploid, were fused to form a diploid. In all cases strains were suitably marked so that diploidy could be confirmed by inspection; e.g. all diploids were non-brown, but were heterozygous with respect to $b w n$, a recessive allele which results in the production of brown pigment. Diploidy was also confirmed by inspecting spore size.

\begin{tabular}{|c|c|c|}
\hline $\begin{array}{l}\text { Name of } \\
\text { diploid }\end{array}$ & $\begin{array}{c}\text { Acriflavin resistance } \\
\text { mutations }\end{array}$ & $\begin{array}{l}\text { Characteristic of } \\
\text { the diploid* }\end{array}$ \\
\hline DP33 & $\operatorname{acr} A 1, \operatorname{acr} A 2$ & $\mathbf{r}$ \\
\hline DP54 & $\operatorname{acr} A I$, acr $A 6$ & $\mathbf{r}$ \\
\hline DP45 & $\operatorname{acr} A I, \operatorname{acr} A 7$ & $\mathbf{r}$ \\
\hline DP55 & $\operatorname{acr} A I$, acr $A 8$ & $\mathbf{r}$ \\
\hline DP46 & $\operatorname{acr} A r, \operatorname{acr} A 9$ & $r$ \\
\hline DP47 & $\operatorname{acr} A I, \operatorname{acr} A I O$ & $\mathbf{r}$ \\
\hline DP8 & $\operatorname{acr} A I,+$ & s \\
\hline DP32 & $\operatorname{acr} A 2,+$ & $\mathbf{s}$ \\
\hline DP44 & $\operatorname{acr} A 6,+$ & s \\
\hline DP59 & $\operatorname{acr} A 8,+$ & $\mathbf{s}$ \\
\hline DP49 & $\operatorname{acr} A 9,+$ & s \\
\hline DP6 & $\operatorname{acr} B_{3}, \operatorname{acr} A I$ & $\mathbf{s}$ \\
\hline DP5 & $\operatorname{acr} B_{3},+$ & s \\
\hline DP35 & $\operatorname{acr} A I, \operatorname{acr} C_{4}$ & $\mathbf{s}$ \\
\hline DP4O & $\operatorname{acrC} 4,+$ & s \\
\hline
\end{tabular}

* r, Resistant, denotes the ability to grow in the presence of acriflavin ( $100 \mu \mathrm{g} / \mathrm{ml})$; s, sensitive, denotes the inability to grow under the same conditions.

\section{RESULTS}

\section{Acriflavin resistance mutations}

Mutations to acriflavin resistance (I00 $\mu \mathrm{g} / \mathrm{ml}$ ) located in two unlinked genes designated acr $A$ and $a c r B$ were isolated and are detailed here.

Isolation of mutants. Strains carrying the allele acr $A$ show cross-resistance to the unrelated compounds methanol $(2 \%, \mathrm{v} / \mathrm{v})$ and thiabendazole (Io $\mu \mathrm{g} / \mathrm{ml}$, gift from Merke, Sharp \& Dohme, New Jersey, U.S.A.). Spontaneous acr $A$ mutants, which were not of clonal origin, were selected at a frequency of approximately $2 \times 10^{-6}$ on nutrient agar plates containing acriflavin (strain NPI4, containing acr $A I$ ), acriflavin followed by reselection on thiabendazole (strain NPI 2, containing acrA2), or methanol (strains NP56, 57, 58, 59, 60, containing acr $A 6$, $7,8,9$, Io respectively).

Only a single mutant of $a c r B$ has been isolated (strain NPI 5, containing $a c r B 3$ ). It is distinguished from acr $A$ mutants by showing no cross-resistance to the other compounds.

Dominance and complementation properties of mutants. Both acr $A$ and acr $B$ mutations are recessive since diploids heterozygous for these resistance markers are sensitive to acriflavin (Table 3), but give rise to acriflavin resistant haploid and diploid clones. Complementation tests on independent mutants containing acr $A$ indicate that they probably involve the same gene, since diploids containing $\operatorname{acr} A I$ with either $\operatorname{acr} A 2,6,7,8,9$, or 10 in trans were all resistant to acriflavin (Table 3). All five strains isolated as resistant to methanol involved a mutation in $\operatorname{acr} A$, hence it is possible that this is the only gene conferring resistance to methanol in $D$. discoideum. Strains containing $\operatorname{acr} B$ (e.g. NPI 5) complement those containing acr $A$ (e.g. NPI4) (Table 3), hence $a c r A$ and $a c r B$ are non-allelic.

Plating efficiency. Strains carrying acr $A$ (e.g. NPI4) give close to I00 $\%$ plating efficiency on nutrient agar plates containing acriflavin or methanol. Growth is slightly slower on plates containing methanol than on nutrient agar alone. Growth of strains containing acr $A$ 


\section{Table 4. Linkage groups in D. discoideum}

The linkage groups have been numbered. In order to standardize nomenclature, we suggest that gene symbols be altered as shown below to conform with the system of Demerec et al. (I966).

\begin{tabular}{|c|c|c|c|c|}
\hline $\begin{array}{l}\text { Linkage } \\
\text { group* }\end{array}$ & $\begin{array}{l}\text { Gene } \\
\text { symbol }\end{array}$ & $\begin{array}{l}\text { Previous } \\
\text { symbol }\end{array}$ & Mutant phenotype & Reference $\dagger$ \\
\hline \multirow[t]{4}{*}{ I } & $c y c A$ & cy ${ }^{r}$ & $\begin{array}{l}\text { Growth in the presence of cycloheximide } \\
(500 \mu \mathrm{g} / \mathrm{ml})\end{array}$ & $\mathbf{I}$ \\
\hline & spr & $\mathrm{sp}^{\mathrm{R}}$ & Round spore & $\mathbf{I}$ \\
\hline & $t s g E$ & $t_{2} s$ & Temperature sensitive for growth & I \\
\hline & $a c r B$ & & $\begin{array}{l}\text { Growth in the presence of acriflavin } \\
(100 \mu \mathrm{g} / \mathrm{ml})\end{array}$ & 2 \\
\hline \multirow[t]{4}{*}{ II } & $w h i$ & $w^{-}$ & White spore & I \\
\hline & $\operatorname{tsg} D$ & $t_{1}{ }^{s}$ & Temperature sensitive for growth & I \\
\hline & $\operatorname{acr} A$ & & $\begin{array}{l}\text { Growth in the presence of acriflavin } \\
(\text { I } 00 \mu \mathrm{g} / \mathrm{ml}) \text { or methanol }(2 \%) \text { or } \\
\text { thiabendazole (Io } \mu \mathrm{g} / \mathrm{ml})\end{array}$ & 2 \\
\hline & axeA & & $\begin{array}{l}\text { Growth in axenic medium (provided that } \\
\text { axe } B \text { is also present) }\end{array}$ & 2 \\
\hline \multirow[t]{2}{*}{ III } & $\operatorname{tsg} A$ & & Temperature sensitive for growth & 3 \\
\hline & $a x e B$ & & $\begin{array}{l}\text { Growth in axenic medium (provided that } \\
\text { axe } A \text { is also present) }\end{array}$ & 2 \\
\hline \multirow[t]{2}{*}{ IV } & $b w n$ & br $^{-}$ & Formation of brown pigment & I \\
\hline & $\operatorname{tsg} B$ & & Temperature sensitive for growth & 3 \\
\hline $\mathrm{V}$ & $\operatorname{tsgC}$ & & Temperature sensitive for growth & 3 \\
\hline
\end{tabular}

on plates containing acriflavin is complicated because some grow poorly (e.g. NP56), while others grow poorly but 30 to $50 \%$ of clones produce vigorous sectors (e.g. NP57, 58, 59, and 60).

Strain NPI 5 bearing $a c r B 3$ has a plating efficiency of $100 \%$ on plates containing acriflavin but produces small colonies when compared with its growth on nutrient agar.

\section{Assignment of acriflavin resistance genes to linkage groups}

$\operatorname{acr} A$. This gene segregates with the associated parental allele at the whi locus and hence has been assigned to linkage group II (Table 4). The allele acr AI was selected in a strain containing the mutant white spore marker (NPI4), and this strain was then fused to form a diploid with an acriflavin sensitive normal yellow spored strain (DPI9: $\frac{\operatorname{acr} A \text { whi }}{+}$ ). In addition, this diploid was heterozygous for markers on linkage groups I ( $c y c A)$, III (tsgA) and IV ( $b w n)$ (Tables I and 2). Subsequent selection on acriflavin or methanol revealed that essentially all haploids had white spores. Haploids with yellow spores were sufficiently rare (4 in 940 segregants screened) to be due to either a mitotic crossover event before or coincident with haploidization (Pontecorvo \& Kafer, I958) or a mutation.

In contrast, when a diploid (e.g. DP44, DP49, DP59) was constructed with acrA in trans to the white spore marker $\left(\frac{a c r A+}{+w h i}\right)$, selection on nutrient agar plates containing acriflavin or methanol produced essentially all yellow haploids, although four white haploids were observed in 565 haploids screened from DP44. Independent segregation of acr $A$ with markers on linkage groups I $(c y c A)$, III $(t s g A)$, IV $(b w n)$, and V $(t s g C)$ was observed. 
$\operatorname{acr} B$. The $\operatorname{acr} B 3$ mutation segregates with the associated parental allele at the $c y c A$ locus and hence has been assigned to linkage group I (Table 4). A diploid (DP5) was constructed with $\operatorname{acr} B_{3}$ in trans with $c y c A I$ and $c i s$ with a spore shape marker and temperature sensitive mutation $\left(\frac{c y c A++++}{+\operatorname{spr} t \operatorname{sg} E \text { acr } B}\right)$. In addition, this diploid was heterozygous for markers on linkage groups II ( $w h i)$ and IV ( $b w n)$ (Tables $\mathrm{I}$ and 2). Selection on nutrient agar plates containing cycloheximide produced only acriflavin sensitive, elliptical spored $\left(s p r^{+}\right)$, temperature resistant $\left(t s g E^{+}\right)$haploids among 67 screened. Selection on nutrient agar plates containing acriflavin produced only cycloheximide sensitive, round spored, temperature sensitive haploids among 92 screened.

Selection on nutrient agar plates containing both cycloheximide and acriflavin produced colonies at considerably less than $\mathrm{I} \%$ the frequency on cycloheximide alone, whereas a diploid (e.g. DP44) with $c y c A$ and acr $A$ in trans $\left(\frac{c y c A}{+} ; \frac{+}{\operatorname{acr} A}\right)$ produced haploid colonies at a frequency between 10 and $50 \%$ of that on cycloheximide alone. Hence $c y c A$ and $a c r B$ are linked, and selection on nutrient agar plates containing both cycloheximide and acriflavin allows the growth of only rare haploids produced by sequential crossover and haploidization events, whereas acr $A$ and $c y c A$ are unlinked and, in theory, $50 \%$ of cycloheximide resistant haploids should also be acriflavin resistant. Independent segregation of acr $B$ with markers on linkage groups II (whi) and IV (bwn) was observed.

\section{Linkage analysis of the ability of strain $\mathrm{Ax} 3$ to grow in axenic medium}

Evidence for the involvement of mutations on linkage groups II and III in the determination of axenic growth in strain $\mathrm{AX}_{3}$ has been obtained. Linkage group II was also implicated in the axenic growth of strain Ax2.

Involvement of linkage group II. When a diploid (e.g. DPI9) was formed from a white spore forming, acriflavin resistant ( $\operatorname{acr} A)$, non-axenic strain, and a yellow-spore-forming, acriflavin-sensitive, axenic strain $\left(\frac{a c r A w h i+}{++ \text { axe }}\right)$, and haploids were selected on nutrient agar plates containing acriflavin, good axenic growers were very rarely obtained. A white, acriflavin-resistant, axenic strain (XII) was obtained only after screening more than 240 white, acriflavin resistant, haploid segregants of DPI9. In contrast, when a diploid (e.g. DP34) was constructed from an acriflavin resistant (acr $A$ ), axenic strain and an acriflavin sensitive, non-axenic strain, such that the axenic genes and acr $A$ were in coupling, then selection on acriflavin or methanol resulted in the recovery of axenic haploids with a high frequency. Of 47 resistant haploid clones, 25 grew axenically while 19 did not, and three were contaminated.

Hence it was suspected that linkage group II (Table 4) contained a gene required for growth in axenic medium. This was confirmed using diploids (e.g. DP8) that were heterozygous for both acr $A$ and whi (linkage group II), and $c y c A$ (linkage group I)

$$
\left(\mathrm{DP} 8: \frac{c y c A}{+} ; \frac{\operatorname{acr} A \operatorname{tsg} D \text { whi }+}{+++a x e}\right) \text {. }
$$

Haploids were selected on cycloheximide and screened for the ability to grow axenically, and also for acriflavin resistance or spore colour markers, or both. This experiment has been repeated a number of times using different diploids, and a representative experiment using DP8 is shown in Table 5(i). No white spore forming, acriflavin resistant haploid grew 
Table 5. Linkage analysis of the ability of derivatives of strain $\mathrm{Ax} 3$ to grow in axenic medium

Only results of selection on nutrient agar plates containing cycloheximide $(500 \mu \mathrm{g} / \mathrm{ml})$ are shown here, since all haploids selected on acriflavin were unable to grow in axenic medium.

(i) $\mathrm{DP} 8=\mathrm{x} 9$ (non-axenic) $\times \mathrm{NP} 3$ (axenic)

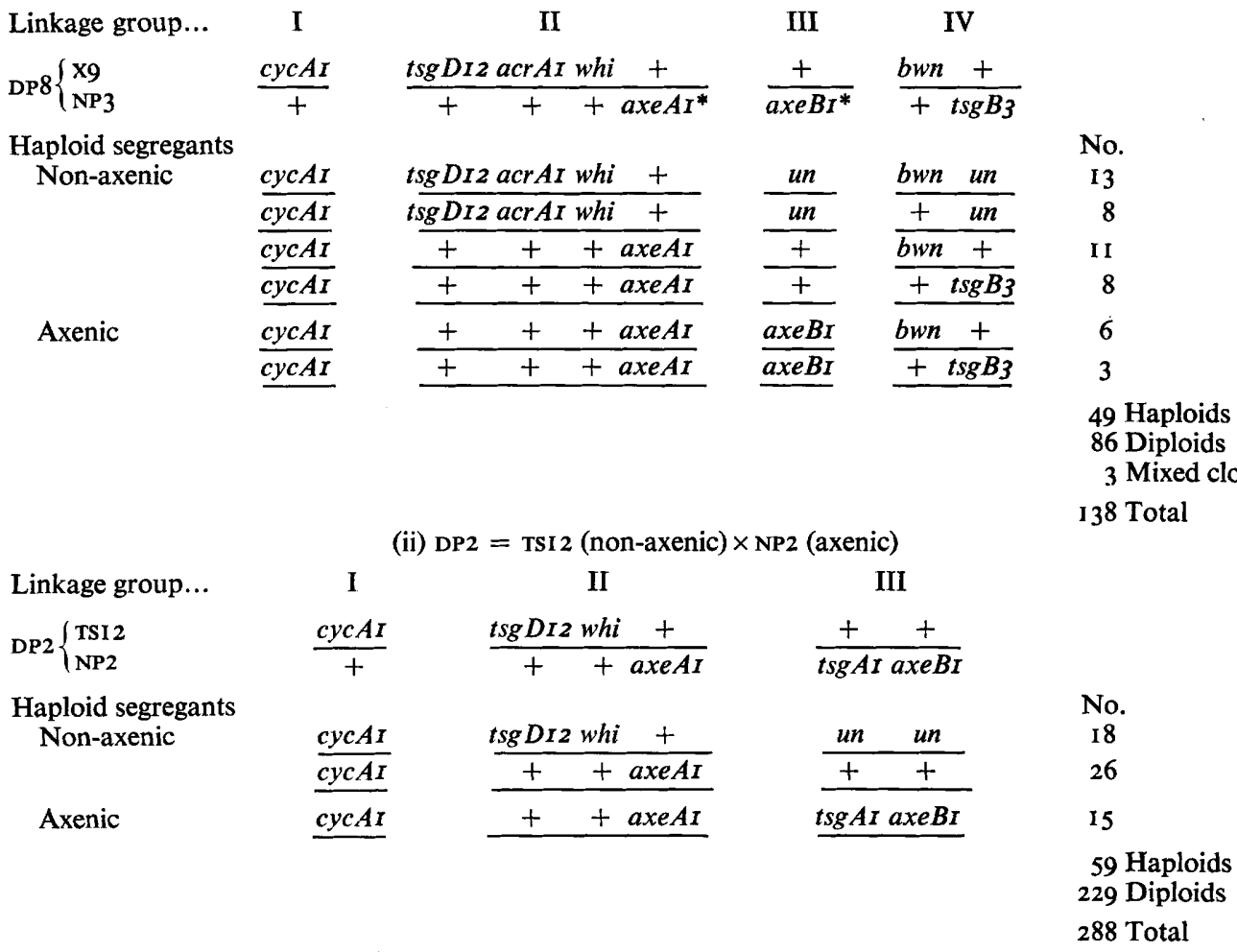

un, Genotype not determined.

* The markers concerning axenic growth (axeA, axeB) represent the proposed axenic genotype; both are required to express the axenic phenotype.

axenically, while about $50 \%$ of the yellow spore forming, acriflavin sensitive haploids were axenic growers.

Linkage group II is also involved in the ability of strain Ax2 to grow axenically, since diploid DP30 $\left(\frac{+}{c y c A} ; \frac{++t \text { axe }}{a c r A \text { tsgD whi }+}\right)$ (Tables I and 2) produced no haploid axenic growers among 125 screened using acriflavin as haploid selector, while yellow, acriflavin sensitive, but not white acriflavin resistant, axenic haploids were commonly recovered using cycloheximide to select segregants. This is not surprising since it has been suggested that the genetic basis of the ability of strains $\mathrm{Ax} 2$ and $\mathrm{AX}_{3}$ to grow axenically may be the same (Williams et al. 1974).

Involvement of linkage group III. Because only about $50 \%$ of the haploids containing linkage group II from an axenic strain ( $\mathrm{Ax} 3)$ grew axenically, it was suspected that a second linkage group was involved in this characteristic. When diploids (e.g. DP2) with the genotype (II, $\frac{t s g D \text { whi }+}{++ \text { axe }}$; III, $\left.\frac{+\quad+}{t s g A \text { axe }}\right)$ were studied, the only haploid axenic strains isolated were 
temperature sensitive and yellow spored. No white spore formers, and no temperature resistant, yellow spored haploids were axenic growers. A representative experiment is shown in Table 5(ii). Hence it was suspected that linkage group III also contained a gene involved in the ability to grow axenically. In subsequent studies only yellow, temperature sensitive axenic strains, or yellow, temperature resistant non-axenic strains have been isolated from diploid DP2, which contains $t s g A I$ from an axenic strain, despite the fact that more than a hundred yellow haploid segregants have been carefully screened. Other diploids gave the same result.

Since linkage groups I, IV (Table 5) and V (unpublished results) from non-axenic strains may be substituted into axenic strains without effect, they do not carry genes for axenic growth. Thus it seems almost certain that for strain $\mathrm{AX}_{3}$ such genes are located only on linkage groups II and III, although until markers are found on the remaining two linkage groups, VI and VII, their involvement cannot be rigorously excluded. The genes for growth in axenic medium on linkage groups II and III have been designated axe $A$ and axeB respectively.

The method used to screen for axenic growth made it difficult to distinguish partial growth (to less than $5 \times 10^{5}$ amoebae $/ \mathrm{ml}$ ) from no growth, so no attempt has been made to determine whether a strain possessing one of the linkage groups II or III derived from an axenic strain has some ability to grow axenically.

\section{Mitotic crossing-over analysis of linkage groups I and II}

Preliminary studies are reported here of homozygous resistant diploids produced from heterozygous, sensitive diploids using selection on nutrient agar plates containing an inhibitor. In Aspergillus nidulans, homozygous diploids are most frequently formed as a result of mitotic crossing-over (Pontecorvo \& Kafer, 1958).

Linkage group $I$. A diploid containing four markers on linkage group I has been constructed for studies on the sequence of markers, DP5 $\left(\frac{\operatorname{acr} B \operatorname{spr} \operatorname{tsg} E+}{+++c y c A}\right)$. All diploids selected on nutrient agar plates containing cycloheximide were homozygous wild type for the other three markers. Diploids selected on nutrient agar plates containing acriflavin were often heterozygous for the other three markers. This result suggests that all four markers are on the same arm of linkage group I, and that $a c r B$ is the most distal, and $c y c A$ the most proximal marker to the centromere. The relative positions of $s p r$ and $t s g E$ have not yet been established.

Linkage group II. The relative positions of three markers on linkage group II are being studied using the diploid DP8 $\left(\frac{a c r A t s g D \text { whi }}{++++} \bigcirc\right)$. Selection of diploids homozygous for acriflavin resistance from DP8 results in three phenotypic classes - white spored, temperature sensitive; yellow spored, temperature sensitive; yellow spored, temperature resistant indicating that the order on the chromosome is $\operatorname{acr} A, \operatorname{tsg} D$, whi, centromere. The results of two other groups investigating the sequence of markers on linkage group II are in agreement with those presented here (E. R. Katz and V. Kao, personal communication; E. B. Gingold and J. M. Ashworth, personal communication).

\section{DISCUSSION}

Acriffavin resistance mutations are proving a profitable source of selective markers for linkage analysis in $D$. discoideum. In addition to the mutants acr $A$ and $\operatorname{acr} B$ described here, 
a third mutant, $\operatorname{acr} C$, which is unlinked to both $\operatorname{acr} A$ and $\operatorname{acr} B$, has been isolated and characterized (F. G. Rothman and E. T. Alexander, personal communication).

In this study the selective markers for cycloheximide and acriflavin (or methanol) resistance have been used to obtain haploid segregants from heterozygous diploids in order to assign the genes for growth in axenic medium to linkage groups. It was assumed that the segregants arose by independent haploidization events, and in many cases I : I segregation of unlinked markers supported this assumption. However, sometimes segregation departed from a $\mathrm{I}: \mathrm{I}$ ratio quite substantially, probably because in these instances many of the segregants were derived from a haploid segregant which arose spontaneously before plating under restrictive conditions. Diploid amoebae of $D$. discoideum spontaneously give rise to haploids (Sussman \& Sussman, 1963; Brody \& Williams, I974). The only way to obtain segregants of certain independent origin is to analyse single segregants from different clones of a heterozygous diploid. This is tedious, and is unnecessary for assigning markers to linkage groups provided that experiments are repeated a number of times using different diploids. For the determination of mitotic map distances (work in progress) it is essential to consider the possible clonal origin of segregants.

Use of the acriflavin resistance mutations $\operatorname{acr} A$ and $\operatorname{acr} B$ has, for the first time in $D$. discoideum, made possible the determination of the sequence of genetic markers on linkage groups II and I respectively, by analysis of mitotic crossing-over. By studying homozygous (resistant) diploids arising from heterozygous (sensitive) diploids under restrictive conditions, the sequence of markers and mitotic map distances can be established. In Aspergillus nidulans such diploids arise (predominantly) as a result of mitotic crossing-over (Pontecorvo \& Kafer, 1958). In the study on D. discoideum by Katz \& Sussman (1972) it was suggested that cycloheximide resistant diploids obtained from a heterozygous (sensitive) diploid on nutrient agar plates containing cycloheximide may have resulted from a non-disjunctional rather than a crossover event, because simultaneous homozygosity for two other markers ( $s p r, t s g E$ ) was observed. We have confirmed that selection of diploids for cycloheximide resistance results in homozygosity for $s p r$ and $t s g E$. However, it appears that this is due to cycloheximide resistance $(c y c A)$ being the most proximal marker to the centromere on linkage group I, since heterozygosity for the markers $c y c A$, $s p r$ and $t s g E$ has been observed when diploids homozygous for $a c r B$ were selected on acriflavin. All of these results are consistent with the suggestion that diploid segregants arise primarily as a result of mitotic crossing-over.

Hence, using genetic methods based on mitotic analysis, both linkage analysis and mapping are now possible in $D$. discoideum.

We thank Professor F. G. Rothman for the gift of strain FGR7 and Mrs D. Berry for technical assistance. R.H.K. is a Damon Runyon post-doctoral fellow. Some of this work was supported by a grant from the Science Research Council. We thank Dr P. J. Piggot and Professor F. G. Rothman for reading the manuscript and for their helpful comments.

\section{REFERENCES}

Brody, T. \& Williams, K. L. (I974). Cytological analysis of the parasexual cycle in Dictyostelium discoideum. Journal of General Microbiology 82, 37I-383.

Clark, M. A., Francis, D. \& Eisenberg, R. (1973). Mating types in cellular slime molds. Biochemical and Biophysical Research Communications 52, 672-678.

Demerec, M., Adelberg, E. A., Clark, A. J. \& Hartman, P. E. (1966). A proposal for a uniform nomenclature in bacterial genetics. Genetics 54, 6I-76. 
Erdos, G. W., RAPER, K. B. \& VoGEN, L. K. (1973). Mating types and macrocyst formation in Dictyostelium discoideum. Proceedings of the National Academy of Sciences of the United States of America 70, 18281830.

Katz, E. R. \& Sussman, M. (1972). Parasexual recombination in Dictyostelium discoideum: selection of stable diploid heterozygotes and stable haploid segregants. Proceedings of the National Academy of Sciences of the United States of America 69, 495-498.

Loomis, W. F. (1971). Sensitivity of Dictyostelium discoideum to nucleic acid analogues. Experimental Cell Research 64, 484-486.

Perkins, D. D. (I962). Preservation of Neurospora stock cultures with anhydrous silica gel. Canadian Journal of Microbiology 8, 59I-594.

Pontecorvo, G. \& Kafer, E. (1958). Genetic analysis based on mitotic recombination. Advances in Genetics 9, $7 \mathrm{I}-\mathrm{IO} 4$.

RAPER, K. B. (1935). Dictyostelium discoideum, a new species of slime mold from decaying forest leaves. Journal of Agricultural Research 5o, I35-147.

Roper, J. A. \& KAFER, E. (1957). Acriflavine-resistant mutants of Aspergillus nidulans. Journal of General Microbiology 16, 660-667.

Schwalb, M. \& Roth, R. (1970). Axenic growth and development of the cellular slime mould Dictyostelium discoideum. Journal of General Microbiology 6o, 283-286.

Sinha, U. \& Ashworth, J. M. (I969). Evidence for the existence of elements of a parasexual cycle in the cellular slime mould Dictyostelium discoideum. Proceedings of the Royal Society B r73, 53 I-540.

Sussman, M. (1966). Biochemical and genetic methods in the study of cellular slime mould development. In Methods in Cell Physiology, vol. 2, pp. 397-410. Edited by D. Prescott. New York: Academic Press.

Sussman, M. \& Sussman, R. R. (1967). Cultivation of Dictyostelium discoideum in axenic medium. Biochemical and Biophysical Research Communications 29, 53-55.

Sussman, R. R. \& Sussman, M. (1963). Ploidal inheritance in the slime mould Dictyostelium discoideum: haploidization and genetic segregation of diploid strains. Journal of General Microbiology 30, 349-355.

Watts, D. J. \& Ashworth, J. M. (1970). Growth of myxamoebae of the cellular slime mould Dictyostelium discoideum in axenic culture. Biochemical Journal 119 , I7I-174.

Williams, K. L., Kessin, R. H. \& Newell, P. C. (I974). Genetics of growth in axenic medium of the cellular slime mould Dictyostelium discoideum. Nature, London 247, 142-143. 